\title{
Lower-Order Aerodynamic Loads Modeling of a Maneuvering Generic Fighter Using DDES Simulations
}

\author{
Tiger L. Jeans ${ }^{1}$ \\ U.S. Air Force Academy, Colorado Springs, CO 80840, USA \\ David R. McDaniel ${ }^{2}$ \\ Air Force Seek Eagle Office, Eglin Air Force Base, Fl 32542, USA \\ Russell M. Cummings ${ }^{3}$, and Keith Bergeron ${ }^{4}$ \\ U.S. Air Force Academy, Colorado Springs, CO 80840, USA
}

\begin{abstract}
Unforeseen nonlinear aerodynamic behavior and/or fluid-structure interactions have affected the development of nearly every major fighter program since 1960. The development cost of each of these aircraft could have been drastically reduced if these aerodynamic issues had been identified earlier in the design process. Therefore, a highfidelity computational tool capable of reliably predicting or identifying configurations susceptible to handling quality instabilities prior to flight test would be of great interest to the stability and control community. The United States Air Force Academy Modeling and Simulation Research Center and the United States Air Force Seek Eagle Office have initiated a joint effort to develop nonlinear lower-order aerodynamic loads models from unsteady CFD solutions. A key step in the process is to perform "training maneuvers," which are dynamic CFD simulations designed to excite the relevant nonlinear flow physics. A reduced-order model is then built using SIDPAC, a regression based modeling technique designed specifically for aircraft system identification. The approach is validated for an aircraft configuration with a known aerodynamic instability that occurs well within the flight envelope. The dynamic CFD simulations can reliably predict this instability for frequencies ranging from 1.43 to $17.1 \mathrm{Hertz}$. In addition, an aerodynamic model trained using a varying frequency chirp maneuver was then used to predict constant frequency aerodynamic loads at conditions where strongly nonlinear aerodynamic behavior occurred.
\end{abstract}

\section{Nomenclature}

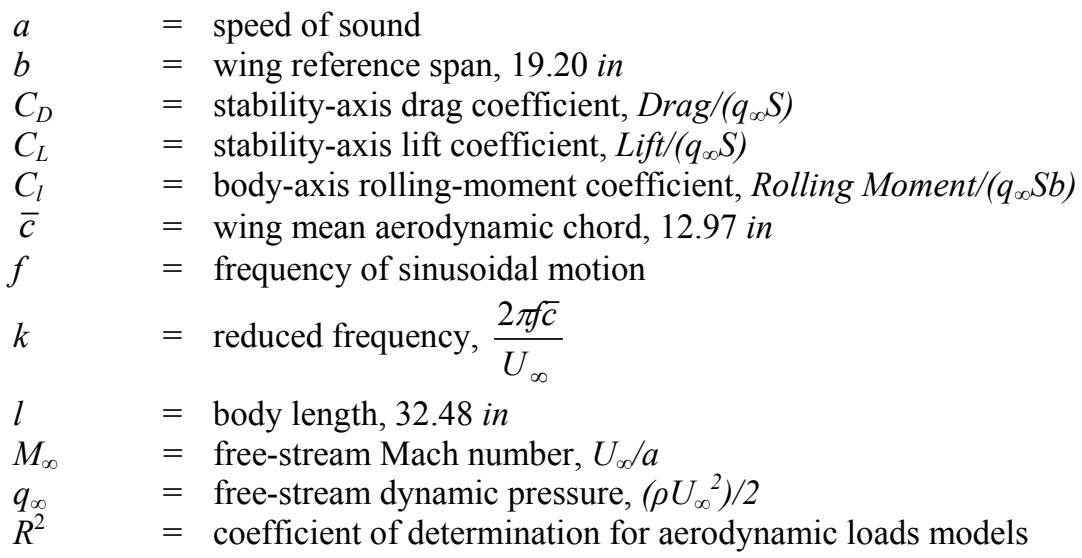

\footnotetext{
${ }^{1}$ Visiting Researcher, Department of Aeronautics, Member AIAA.

${ }^{2}$ Aerospace Engineer, Air Force Seek Eagle Office, Member AIAA.

${ }^{3}$ Professor, Department of Aeronautics, Associate Fellow AIAA.

${ }^{4}$ Director M\&SRC, Department of Aeronautics, Member AIAA.
} 


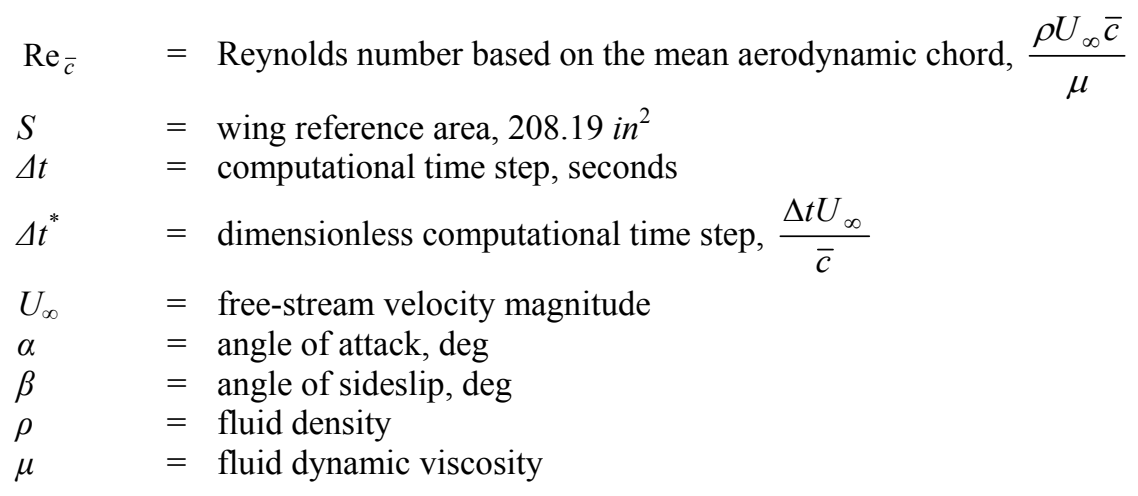

\section{Introduction}

$\mathrm{H}$ istorcially, Stability and Control $(\mathrm{S} \& \mathrm{C})$ engineers have used an iterative process combining semi-empirical lower-order, wind-tunnel, and flight test modeling techniques to determine the aerodynamic characteristics of new fighter aircraft. Despite their greatest efforts using the best available predictive capabilities, nearly every major fighter program since 1960 has had costly nonlinear aerodynamic or fluid-structure interaction issues that were not discovered until flight testing. ${ }^{1}$ Some examples include the F-15, ${ }^{2} \mathrm{~F} / \mathrm{A}-18 \mathrm{~A},{ }^{2} \mathrm{~F} / \mathrm{A}-18 \mathrm{C},{ }^{3} \mathrm{AV}-8 \mathrm{~B},{ }^{2}$ and the B-2 Bomber. $^{4}$ The F-15, F/A-18A, and AV-8B all exhibited significant aero-elastic flutter, ${ }^{2}$ while the F/A-18C experienced tail buffet at high angles of attack due to leading-edge extension vortex breakdown, ${ }^{3}$ and the B-2 Bomber experienced a residual pitch oscillation. ${ }^{4}$ The development costs of each of these aircraft could have been drastically reduced if these issues had been identified earlier in the design process. However, existing semiempirical lower-order modeling and wind-tunnel techniques are incapable of reliably predicting unsteady nonlinear aerodynamic behavior over the full flight envelope. Clearly, a high-fidelity computational tool capable of reliably predicting and/or identifying configurations susceptible to handling quality instabilities prior to flight testing would be of great interest to the S\&C community. Such a tool is well suited to the aircraft design phase and would decrease the cost and risks incurred by flight-testing and post-design-phase modifications. In addition, there is an immediate need for this capability in the test and evaluation community. In particular, the Air Force Seek Eagle Office (AFSEO) would integrate this tool into their store certification process for existing aircraft. Currently, AFSEO has no computational method for certifying the flying and handling qualities of new configurations and must resort to risky semi-empirical methods, or costly (approximately $\$ 100 \mathrm{~K} / \mathrm{hr}$ ) wind-tunnel and flight testing techniques with lengthy lead times (up to one year).

In response to this need, recent research at AFSEO and the United States Air Force Academy (USAFA) has focused on improving nonlinear lower-order aerodynamic loads S\&C modeling techniques. With recent advances in computational techniques, turbulent Navier-Stokes solvers are now capable of capturing the unsteady nonlinear aerodynamic behavior that leads to the various static and dynamic instabilities mentioned above. . $^{5-12}$ As such, the focus of the S\&C research has been to effectively incorporate Computational Fluid Dynamics (CFD) into the model development process using dynamic CFD solutions of complete aircraft configurations. The ultimate goal is to develop a methodology for efficiently and accurately screening for nonlinear aerodynamic phenomena such as spin, tumble, lateral instabilities, limit-cycle oscillations, and tail buffet of full aircraft. This has lead to an innovative approach for modeling aircraft S\&C characteristics, the details of which can be found in McDaniel and Morton. ${ }^{1}$ A schematic of their approach is graphically represented in Figure 1, and a summary is as follows: (a) CFD simulations are performed using computational training maneuvers designed to excite the relevant flow physics encountered during actual missions, (b) a mathematical Reduced Order Model (ROM) is built of the aircraft response using system identification methods, (c) the model is validated by comparing CFD simulations against model predictions, and (d) predictions of all flight test points are made using the model to determine the expected behavior of the aircraft. This process would identify unexpected S\&C issues early in the design process. 
Matthews and Schwartz ${ }^{13}$ showed that determining the appropriate training maneuver that properly excites all the relevant flow physics is not a trivial task but is vital to the success of the final aerodynamic model. Early work indicated that chirp signals were the best candidate for training the reduced-order model. ${ }^{14}$ However, the complex relationship between the computational training maneuver and resulting ROM is not yet fully understood, and further basic research is necessary for the computational tool to successfully reach its full potential. In particular, a fundamental study is needed to develop more reliable and robust techniques for ROM interpolation. In particular, new approaches must be developed for interpolating between the independent variables of the $\operatorname{ROM}(\alpha, \beta, p, q, r$, etc.) within the nominal parameter ranges of the training maneuver at a particular Mach/altitude, and between different Mach/altitudes at specified parameters within the training maneuver. In addition, to date the research has focused on aircraft with fairly benign aerodynamic characteristics and therefore, the above methodology must be verified and validated for aircraft with nonlinear aerodynamic behavior. As such, the primary objective of this paper is to validate the modeling approach outline above for an aircraft configuration with a known aerodynamic instability that occurs well with the flight envelope. A secondary objective is to improve the current understanding between the selected training maneuver and resulting ROM, in particular, for aircraft with nonlinear aerodynamic behavior.

The generic fighter chosen for this study was the Modular Transonic Vortex Interaction (MTVI), which was developed at the NASA Langley Research Center. ${ }^{15-17}$ The MTVI has a chine forebody-delta wing configuration and was initially developed to study the interaction of the chine and wing leading-edge vortices. It is well known that the chine generally results in stronger forebody vortices being shed compared to traditional fighters with smooth forebodies. For certain flight conditions these vortices interact with the wing leading edge vortices, and improve the maneuvering lift capabilities. ${ }^{18}$ However, for other flight conditions these vortex interactions are detrimental to the aerodynamic characteristics of the vehicle. This is especially true when the fighter experiences moderate to low angles of sideslip at angles of attack in excess of $25^{\circ}$. Under these conditions abrupt asymmetric vortex breakdown leads to pronounced pitch-up and significant nonlinearities in lateral stability that could result in roll departure. ${ }^{15}$

The MTVI configuration has been previously investigated both experimentally ${ }^{15-18}$ and computationally. ${ }^{12,}$ 19-27 In particular, Hall ${ }^{18}$ experimentally studied the impact of the fuselage cross-section on the stability of the MTVI body shape without employing leading edge flaps or vertical tails. He tested a $30^{\circ}$ and $100^{\circ}$ chine cross-section in the upright and inverted positions. These experiments were conducted at Mach $=0.4$ for Reynolds numbers ranging from $2.61 \times 10^{6}$ to $2.84 \times 10^{6}$. Hall found significant nonlinearities in the rolling moment for all cross sections for $\alpha$ $\geq 23^{\circ}$ and $-5^{\circ}<\beta<5^{\circ}$. Jeans et al. ${ }^{12}$ utilized Delayed Detached-Eddy Simulation (DDES) to computationally predicted, for the first time, the roll instability measured by Hall for the $30^{\circ}$ chine cross-section at $\alpha=30^{\circ}$. It is this large instability that makes the MTVI a perfect test configuration for validating new low-order aerodynamic loads model concepts.

The MTVI configuration employed for this study utilized the $30^{\circ}$ chine fuselage. A schematic of the overall shape along with the fuselage cross-section are shown in Fig. 2. The MTVI body has a span of 29.90 inches and a length of 32.48 inches. The wing is comprised of a cropped-delta planform with a $60^{\circ}$ leading edge sweep and a NACA 65-005 airfoil section modified with double-arc section forward of the maximum thickness and sharp leading edges. ${ }^{15}$ Non-dimensional forces and moments are calculated relative to the standard body axis system with the origin located at the quarter chord of the mean aerodynamic chord, which is 20.36 inches from the nose. 


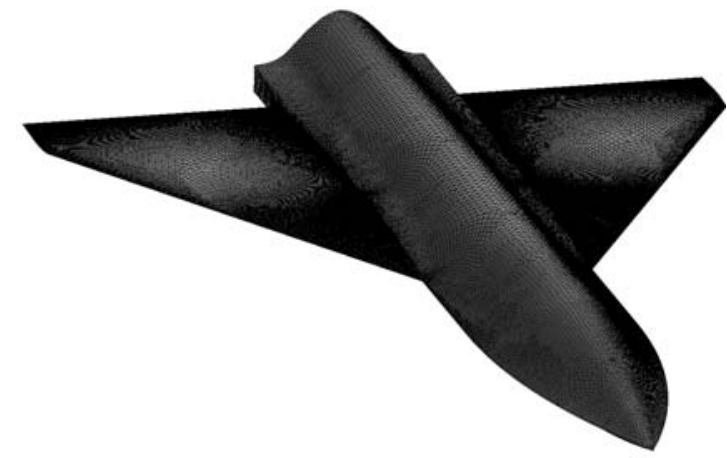

(a)

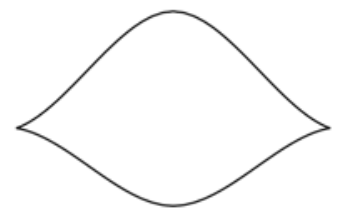

(b)

Figure 2. (a) Schematic of overall MTVI configuration and surface mesh. (b) Schematic of forebody cross section with $30^{\circ}$ chine.

\section{Computational Methodology}

\section{A. Flow Solver}

Computations were performed using the commercially available flow solver Cobalt, ${ }^{28}$ which solves the unsteady, three-dimensional, compressible Navier-Stokes equations. Cobalt is a cell-centered, finite volume based code applicable to arbitrary cell topologies including prisms, tetrahedra and hexahedrals. Second-order accuracy in space is achieved using the exact Riemann solver of Gottleib and Groth, ${ }^{29}$ and least squares gradient calculations using QR factorization. To advance the discretized system a point-implicit method using analytic first-order inviscid and viscous Jacobians is used. A Newton sub-iteration method is used in the solution of the system of equations to improve time accuracy of the point-implicit method. The method is second-order accurate in time. Tomaro et al. ${ }^{30}$ converted the code from explicit to implicit, enabling CFL numbers as high as $10^{6}$.

Time-dependent dynamic computations were performed using the DDES technique proposed by Spalart et al. ${ }^{31}$ Cobalt has been previously used in conjunction with both DES and DDES to successfully model similar high angle of attack flows. $.^{6-9}, 11-12$ DDES is conceptually similar to the original DES technique proposed by Spalart et al. ${ }^{32}$ where the entire boundary layer is treated using a Reynolds-Averaged Navier-Stokes (RANS) model and highly separated regions are treated using Large Eddy Simulation (LES). This results in a numerically feasible approach that combines the most favorable elements of each method. Both the original DES and DDES models are based on the Spalart-Allmaras ${ }^{33}$ (SA) one-equation RANS turbulence model. The primary difference between DES and DDES is the formulation of the limiter used to transition between RANS and LES turbulence treatment, where the DDES formulation is intended to remove the ambiguous-grid issue emphasized by Menter and Kuntz. ${ }^{34}$ In the original DES formulation the limiter depended only on the grid, but in the current DDES formulation the limiter depends on the eddy-viscosity field. It should also be noted that for the current study the Spalart-Allmaras Rotational Correction ${ }^{35}$ (SARC) turbulence model was implemented. The SARC turbulence model includes modifications to the original SA model to account for the effect of system rotation and/or streamline curvature.

Cobalt is cast in an arbitrary Lagrangian-Eulerian formulation, and therefore, has the ability to compute rigidbody motion. ${ }^{36}$ Cobalt has both free and specified 6-degree-of-freedom rigid-body motion capabilities. Rigid-body motion reorients the grid without deforming it using a transformation matrix and a translation vector describes the motion of the grid.

\section{B. Mesh Generation}

The mesh used for the current study was created using Solidmesh $3 \mathrm{D},{ }^{37}$ which is an Advancing Front Local Reconnection (AFLR) unstructured grid generator. An unstructured triangular mesh was generated over the surfaces of the domain and volume elements were then inflated. An example of the surface mesh on the MTVI body surface is shown in Fig. 2 (a). The first node height was chosen using the approach of Cummings et al. ${ }^{38}$ such that the average $\mathrm{y}^{+}$was below 0.5 , which ensured an adequate volume mesh that correctly models the boundary layer. The final mesh chosen for this study consisted of 10.9 million cells. A cross sectional view of the mesh elements at $\mathrm{x} / l=$ 0.88 and a side view of the mesh near the y-z plane are shown in Figures 3 (a) and (b), respectively. As shown in Fig. 3 (a), planar mesh controls were implemented above the body such that elements were clustered just above the fuselage and wing to ensure that the forebody and leading edge vortices were properly convicted downstream.

4

American Institute of Aeronautics and Astronautics

DISTRIBUTION A. Approved for public release, distribution unlimited. 


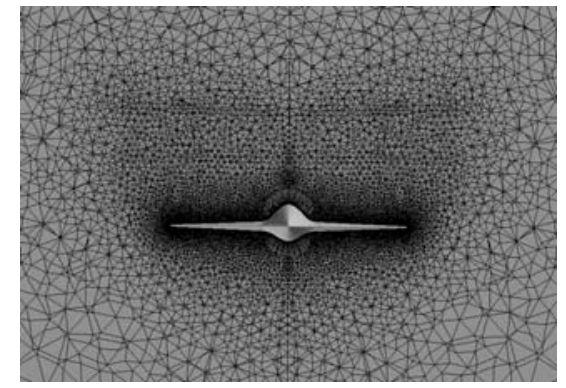

(a)

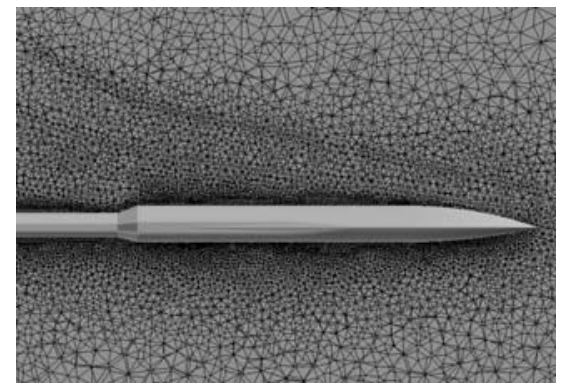

(b)

Figure 3. (a) Cross sectional view ( $x-y$ plane) of the medium density mesh at $x / l=0.88$. (b) Side view of the medium density mesh at $\mathrm{y} / \mathrm{l}=\mathbf{0 . 0 6}$.

\section{Computational Setup}

The computational domain was rectangular in shape with the MTVI geometry centrally located. The minimal distance from the body to each of the outer boundaries was $10 \mathrm{l}$. The no-slip adiabatic wall boundary condition was employed for the body surface and the modified Riemann-invariant condition was implemented at the farfield boundary. It should also be noted that a sting matching the one used in the wind tunnel experiments ${ }^{15-17}$ was incorporated into the geometry. The no-slip adiabatic wall condition without force accounting was implemented on the sting.

Time-dependent computations were performed at $\operatorname{Re}_{\bar{c}}=2.68 \times 10^{6}, M_{\infty}=0.40$ and $\alpha=30^{\circ}$. The solution was initialized using a static solution at $\alpha=30^{\circ}$ and $\beta=0^{\circ}$, and the aircraft was yawed about the wind axis in a sinusoidal manner between $\beta=-5^{\circ}$ to $\beta=5^{\circ}$ at various frequencies. Further details of the dynamic maneuvers are presented in Section III. To ensure that the flow solution was converged at every time step, five Newton sub-iterations were used.

\section{Grid Refinement and Time Step Study}

Cummings et al. ${ }^{38}$ showed that for time-dependent flows a joint time step/grid density study is required, because different meshes obtain time independence at different time step values. They developed a method for ensuring that both temporal and spatial independence have been achieved, which is based on analyzing the frequency content of an appropriate flow field variable (e.g. integrated forces or moments or pressures at specific locations) using Power Spectrum Density (PSD) analysis.

A comprehensive grid/time step study was performed for static simulations at $\alpha=30^{\circ}$ and $\beta=0^{\circ}$ using the method developed by Cummings et al., ${ }^{38}$ and the results were presented in Ref. 12. Simulations were completed using three meshes ranging in density from 5.5 million to 22.6 million cells and dimensionless time steps ranging from $\Delta t^{*}=0.08$ to $\Delta t^{*}=0.005$, where $\Delta t^{*}$ is defined by,

$$
\Delta t^{*}=\frac{\Delta t U_{\infty}}{\bar{c}} .
$$

To determine the flow field frequency content, a PSD analysis of pitching moment was completed for each solution. The conclusion from this study was that the solutions are spatially and temporally converged using the 10.9 million cell mesh in conjunction with $\Delta t^{*}=0.01$. It was then assumed that these parameters were also sufficient for the dynamic simulations.

\section{Stability and Control Aerodynamic Loads Modeling}

System IDentification (SID) is the process of constructing a mathematical model from system input and output data, and then characterizing both the system uncertainties and noise. ${ }^{39}$ The results from the CFD simulations represent the computational training maneuver and will be used as input into the SID process. Morelli developed a global nonlinear parameter modeling technique based on regression methods specifically for aircraft applications known as System IDentification Program for AirCraft (SIDPAC). ${ }^{40,41}$ This technique is used to estimate the functional relationship between the independent variables of the aircraft motion $(\alpha, \beta, p, q, r$, etc.) and the computed 
aerodynamic loads. The resulting SIDPAC model will then be used to interpolate aerodynamic loads within the parameter space.

\section{Results and Discussion}

\section{A. Static Simulations}

A detailed investigation of the static simulations can be found in Jeans et al. ${ }^{12}$ A limited subset of these results is presented here primarily to provide a complete understanding of the flow field and for comparison to the dynamic results. The complete dataset includes an angle of attack sweep from $\alpha=10^{\circ}$ to $30^{\circ}$ at zero sideslip, and angle of sideslip sweep from $\beta=0^{\circ}$ to $5^{\circ}$ at $\alpha=30^{\circ}$.

The primary means of validating the DDES predictions is by comparison of overall static force and moment coefficients with the experimental data in Ref. 18. Timeaveraged DDES predictions of lift and drag at $\alpha=30^{\circ}$ and $\beta=0^{\circ}$ are $C_{L}=1.616$ and $C_{D}=0.910$, which correspond to a percent difference of $0.13 \%$ and $1.8 \%$, respectively, compared to experimental data. A comparison of DDES predictions and experimentally measured rolling moment coefficients as a function of $\beta$ is shown in Fig. 4. To highlight the hysteresis in the experimental data both positive and negative sideslip are plotted on the same axis. The most significant feature in this figure is the abrupt nonlinear change in rolling moment between $\beta=1^{\circ}$ and $2^{\circ}$. Although it was not presented in the experimental dataset, the DDES simulations also predict approximately a $16 \%$ decrease in both lift and drag between $\beta=1^{\circ}$ and $2^{\circ}$ (see Fig. 5).

Major vortical structures are highlighted in Fig. 6 using the $\mathrm{Q}$ vortex identification criterion. ${ }^{42}$ Plotted are isosurfaces of $\mathrm{Q}=1.0 \times 10^{7} \mathrm{~s}^{-2}$ at $30^{\circ}$ incidence at $\beta=1.0^{\circ}$ and $\beta=2.0^{\circ}$. It should be noted that the left side of each figure is the windward side of the body. The primary fuselage and leading edge vortices are clearly evident in both figures. The principal difference between Fig. 6 (a) and (b) is the breakdown of the vortical structures over the windward wing at $\beta=2.0^{\circ}$. It was shown in Jeans et al. ${ }^{12}$ that this asymmetric vortex breakdown was responsible for the significantly nonlinear behavior of rolling moment curve in Fig. 4. This was largely due to an abrupt and significant change in the surface pressure distribution on the top windward wing.

\section{B. Dynamic Simulations}

All of the dynamic CFD simulations were performed at the Arctic Region Supercomputing Center (ARSC) on Midnight, a Sun cluster comprised of 2312 Opteron processors with a 68 TB Lustre file system.

\section{Motion Properties}

All of the prescribed maneuvers were completed at a constant incidence angle of $\alpha=30^{\circ}$ and the sideslip angle

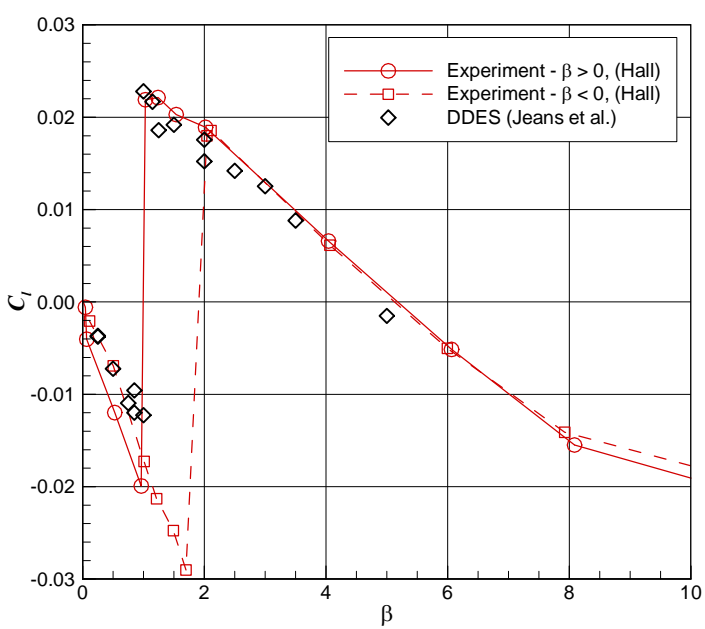

Figure 4. A comparison of CFD predictions and experimental static rolling moment coefficients as a function of sideslip angle for $\alpha=30^{\circ}, M_{\infty}=$ 0.4 , and $\operatorname{Re}_{\bar{c}}=2.68 \times 10^{6}$.

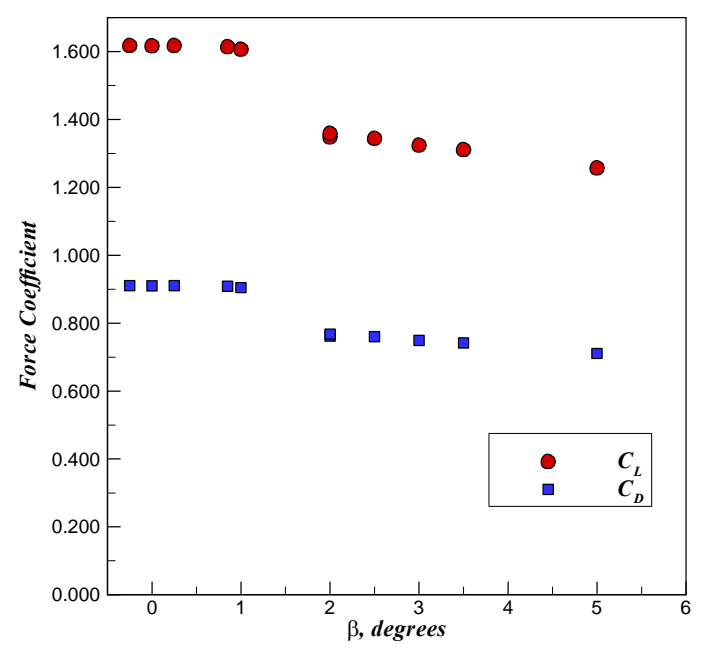

Figure 5. DDES predictions of static lift and drag coefficient as a function of sideslip angle for $\alpha=30^{\circ}, M_{\infty}=0.4$, and $\operatorname{Re}_{\bar{c}}=2.68 \times 10^{6}$.
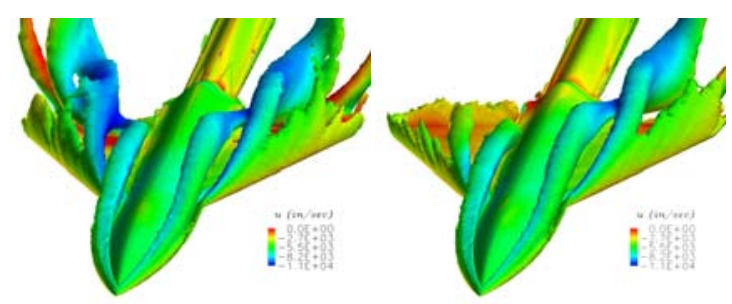

(a) $\beta=1^{\circ}$

(b) $\beta=2^{\circ}$

Figure 6. Isosurfaces of $Q=1.0 \times 10^{7} \mathrm{~s}^{-2}$ for static DDES simulations at $\alpha=30^{\circ}$ and $\beta=1^{\circ}$ and $2^{\circ}$. The isosurface is colored by axial velocity with red corresponding to $u=0$ and blue corresponding to $u=-2 u_{\infty}$. 
was varied in a sinusoidal manner from $\beta=5^{\circ}$ to $\beta=-5^{\circ}$. A total of six simulations were included in the final dataset, five varied the sideslip angle at a constant frequency and one chirp motion varied the frequency in a linear fashion. The five constant frequency motions were completed at $k=0.021,0.042,0.085,0.170$, and 0.255 , where $k$ is the reduced frequency defined as,

$$
k=\frac{2 \pi f \bar{c}}{U_{\infty}}
$$

This corresponds to frequency values of $f=1.43$, 2.85, 5.7, 11.4, and 17.1 Hertz, respectively. The chirp motion reduced frequency varied linearly from $k_{\min }=0$ to $k_{\max }=0.089$.

Plotted in Fig. 7 is sideslip angle as a function of time for the constant frequency motion at $k=0.042$ and chirp maneuver. Two complete periods were completed for the CFD simulation at $k=0.042$ and the results showed that the body forces and moments from the first period were repeated during the second period. Therefore, to minimize the required CPU hours, all other constant frequency simulations were terminated once the predicted body forces and moments began to repeat.

Regressor maps for reduced body-axis rolling moment, $\hat{p}$, and yawing moment, $\hat{r}$, as a function of sideslip angle are shown in Fig. 8 for each motion, where $\hat{p}$ and $\hat{r}$ are defined as,

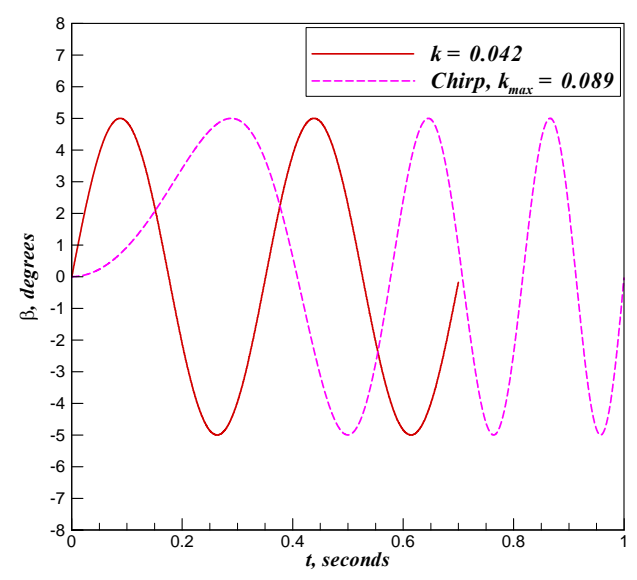

Figure 7. Sideslip angle as a function of time for constant frequency motion at $k=0.042$ and chirp maneuver.

$$
\hat{p}=\frac{p b}{2 U_{\infty}} \text { and } \hat{r}=\frac{r b}{2 U_{\infty}}
$$

The dynamic parameters are reported in reduced quantities so that they can be compared to maximum allowable values for a typical full-scale fighter aircraft. The maximum rolling moment varied from $\hat{p}=0.0005$ at $k=0.021$ to $\hat{p}=0.008$ at $k=0.255$, and the maximum yawing moment varied from $\hat{r}=0.001$ at $k=0.021$ to $\hat{r}=0.014$ at $k=$ 0.255 . For comparison, the maximum reduced rolling and yawing moments allowable for the F-16 are on the order of $\hat{p}=0.2$ and $\hat{r}=0.009$.

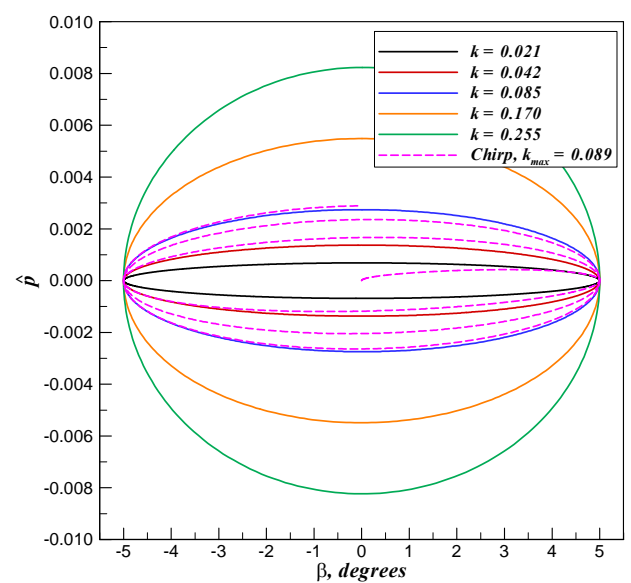

(a) Rolling moment.

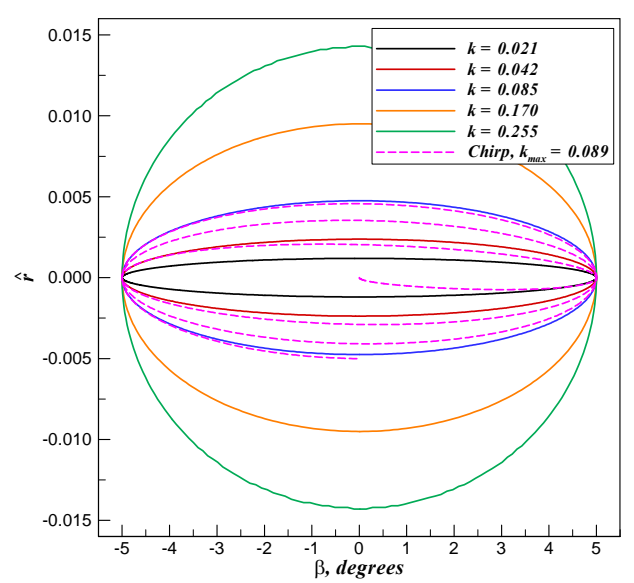

(b) Yawing moment.

Figure 8. Reduced rolling and yawing moment as a function of sideslip angle for all of the maneuvers.

7

American Institute of Aeronautics and Astronautics

DISTRIBUTION A. Approved for public release, distribution unlimited. 


\section{Constant Frequency Results}

This section describes the predicted aerodynamic characteristics of the constant frequency motion simulations. The overall body force and moment coefficient are presented first, followed by a detailed description of the flow field.

Dynamic lift, drag, and rolling moment predictions are plotted in Fig. 9 as a function of sideslip angle for each of the constant frequency simulations. Also plotted are the static experimental and CFD predictions. The lift and drag predictions in Fig. 9 (a) have very similar topologies, and therefore are discussed concurrently. During the initial upstroke from $\beta=0^{\circ}$ both lift and drag remain constant at the static value for $\alpha=30^{\circ}$ and $\beta=0^{\circ}$. At the lower frequencies $(k=0.021$ and 0.042$)$, as sideslip continues to increase both lift and drag quickly decline such that at $\beta=$ $5^{\circ}$ both simulations are in agreement with the static values. As the motion is reversed and sideslip angle declines from $\beta=5^{\circ}$, both simulations predict a linear increase in lift and drag that initially follows the static predictions. However, a key difference from the static predictions is that this linear increase is sustained until $\beta=-3^{\circ}$ at $k=$ 0.021 and $\beta=-4^{\circ}$ at $k=0.042$, at which point there is a second rapid decrease in both lift and drag. As the motion is reversed and sideslip angle is increased from $\beta=-5^{\circ}$, both simulations predict a second linear increase in lift and drag that again follows the static predictions, but continues until $\beta=3^{\circ}$ at $k=0.021$ and $\beta=4^{\circ}$ at $k=0.042$. At this point the force predictions begin to repeat. As frequency increased from $k=0.085$ to $k=0.255$, the rapid decrease in lift and drag experienced at the lower frequencies is less significant such that at $k=0.255$ the body forces are nearly horizontal and independent of sideslip.

Of greater interest to the current research are the dynamic rolling moment coefficients plotted in Fig. 9 (b). Note that the shape of the dynamic curves is very different from the static curve. At the lower frequencies $(k=0.021$ and 0.042 ), the nonlinearity is delayed until greater sideslip angles and when the motion is reversed the nonlinearity is delayed until negative sideslip angles. This results in a general topology that resembles a tilted rectangular box rather than the m-shaped static curve. As the frequency increases the nonlinearity is reduced and the data collapses such that at $k=0.17$ the dynamic curve is nearly linear for the full range of sideslip angle. Therefore, it is at the lower frequencies that the aircraft experience the most drastic nonlinear aerodynamic behavior.
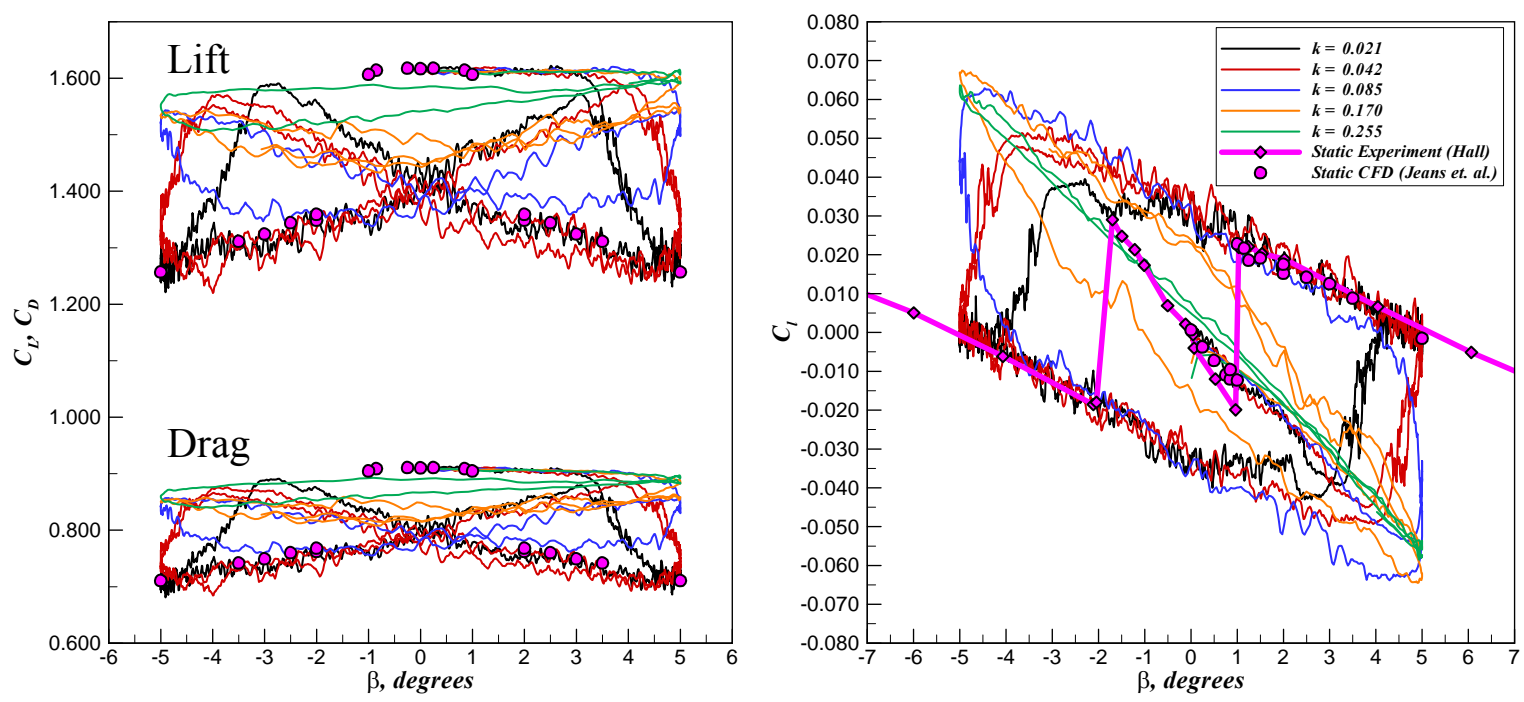

(a) Lift and drag coefficient (see (b) for legend). (b) Rolling moment coefficient.

Figure 9. CFD predicted dynamic lift, drag, and rolling moment coefficient as a function of sideslip angle for $\alpha=30^{\circ}, M_{\infty}=0.4$, and $\operatorname{Re}_{\bar{c}}=2.68 \times 10^{6}$.

To aid with a detailed description of the rolling moment curves in Fig. 9 (b), major vortical structures are highlighted in Figs. 10 and 11 using the Q vortex identification criterion. It should be noted that in Fig. 10 the data was outputted in the grid reference frame and therefore the vehicle appears stationary, whereas, in Fig. 11 the data was outputted in the laboratory reference frame and therefore the vehicle appears to move with the specified motion.

Plotted in Fig. 10 are isosurfaces of $Q$ at specific frames for the low frequency simulations $(k=0.021$ and 0.041$)$. Frame a1-a9 correspond to $k=0.021$ and frames b1-b3 correspond to $k=0.042$. All of the simulations are restarted from the static $\alpha=30^{\circ}, \beta=0^{\circ}$ simulation, and therefore the flow is initially symmetric with well developed vortices as shown in frame a1. As previously mentioned, during the initial upstroke the dynamic rolling moment predictions

8

American Institute of Aeronautics and Astronautics

DISTRIBUTION A. Approved for public release, distribution unlimited. 
follow the static data. However, unlike the static data, the rolling moment continues to decrease linearly with sideslip angle until $\beta=3^{\circ}$ for $k=0.021$ and $\beta=4^{\circ}$ for $k=0.042$. As shown in frames a2 and $\mathrm{b} 1$, this is because the dynamic motion delays the windward vortex breakdown. As sideslip angle is increased further (frames a3, a4, and b2), the windward vortex system breaks down, resulting in an abrupt nonlinear increase in rolling moment that is similar to the static data. Once the maximum sideslip angle is reached $\left(\beta=5^{\circ}\right)$ and the down stroke is initiated, the rolling moment increases linearly such that there is a significant positive rolling moment at $\beta=0^{\circ}$. As shown in frame a5, this is because the windward vortex system does not reform during the down stroke, which is a key difference from the static data. This flow topology continues until $\beta=-3^{\circ}$ for $k=$ 0.021 and $\beta=-4^{\circ}$ for $k=0.042^{\circ}$, at which point the leeward vortex system re-forms and the windward vortex system breaks down (frames a6, a7, and b3) resulting in an abrupt nonlinear decrease in rolling moment that is similar to the static data. Once the minimum slide angle is reached $\left(\beta=-5^{\circ}\right)$ and the upstroke is initiated, the rolling moment decreases linearly such that there is a significant negative rolling moment at $\beta=0^{\circ}$. As shown in frames a8 and $a 9$, this is because the windward vortex system does not reform during the upstroke, which is the second key difference from the static data. This flow topology continues until $\beta=3^{\circ}$ for $k=$ 0.021 and $\beta=4^{\circ}$ for $k=0.042^{\circ}$, at which point the leeward vortex system reforms, the windward vortex system breaks down, and the rolling moment predictions begin to repeat. By comparison of frames b1-b3 to a1-a9, one can conclude that the primary difference between $k=0.021$ and $k=0.042$ is that at $k=0.042$ the vortex breakdown is delayed until greater positive and negative sideslip angles.

Plotted in Fig. 11 are isosurfaces of Q at specific frames for the high frequency simulations $(k=0.17$ and 0.255$)$. Frame a1-a6 correspond to $k=0.17$ and frames b1-b3 correspond to $k=0.042$. As shown in frames a2-a5, windward vortex breakdown still occurs at $k=0.17$, however it is delayed until the sideslip
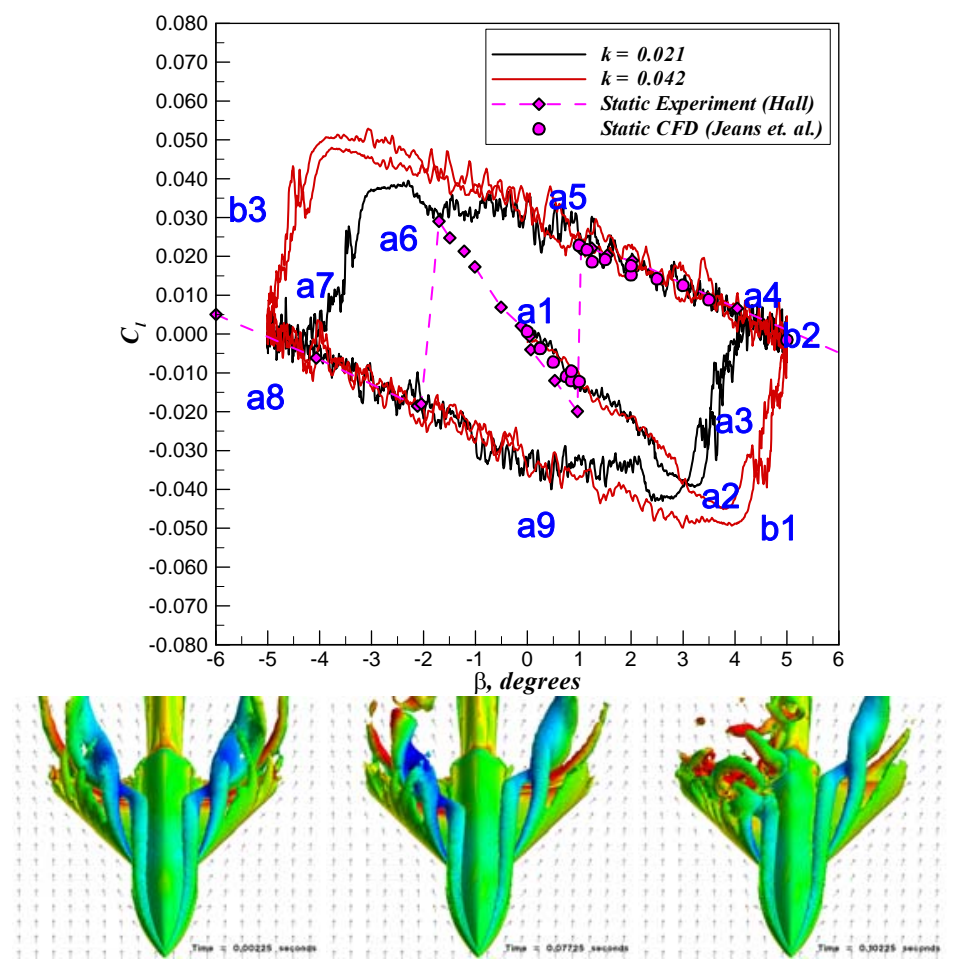

a1

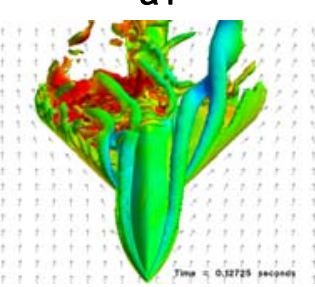

a2

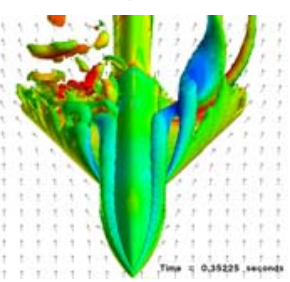

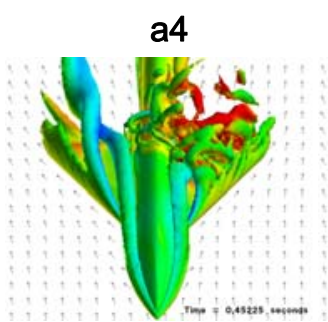

a7

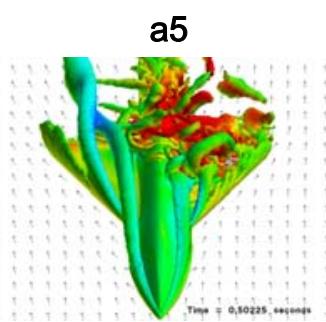

a8 a3

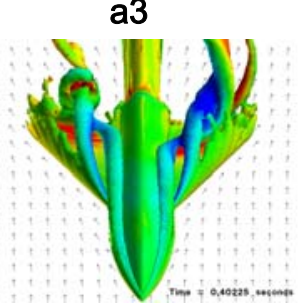

a6 (a) $k=0.021$.

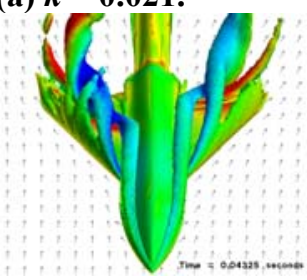

b1

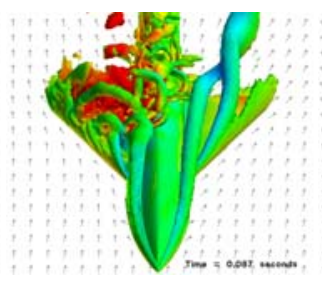

b2

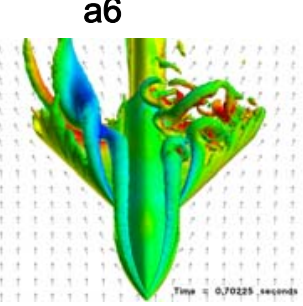

a9 (b) $k=0.042$.

Figure 10. Isosurfaces of $Q=1.0 \times 10^{7} \mathrm{~s}^{-2}$ at selected frames for low frequency simulations at $k=0.021$ and $k=0.042$. Isosurfaces are colored by axial velocity with red corresponding to $u=0$ and blue corresponding to $u=-2 u_{\infty}$.

9

American Institute of Aeronautics and Astronautics DISTRIBUTION A. Approved for public release, distribution unlimited. 
angle has reached its maximum or minimum value. In addition, the vortex breakdown is much less severe compared to the lower frequency simulations, thereby reducing the severity of the nonlinear behavior in rolling moment. As shown in frames b1-b3, when the frequency is increased to $k=0.255$ the windward vortex no long fully breaks down, thereby completely removing the nonlinear aerodynamic behavior in rolling moment.

\section{Chirp Motion Results}

This section describes the aerodynamic characteristics of the chirp training maneuver. Dynamic lift, drag, and rolling moment predictions are plotted in Fig. 12 as a function of sideslip angle. Also plotted are the experimental and CFD static predictions. As shown in Fig. 8, the regressor map of the chirp maneuver bounds the constant frequency simulations at $k=0.021,0.042$ and 0.085. A comparison of the force and moment predictions to the constant frequency data in Fig. 9 show that the chirp maneuver exhibits characteristics similar to all three constant frequency simulations. Initially, the rolling moment predictions are similar to the $k=0.21$ simulation, and as the frequency increases the rolling moment predictions transition such that they are similar to the $k=0.085$ simulation.
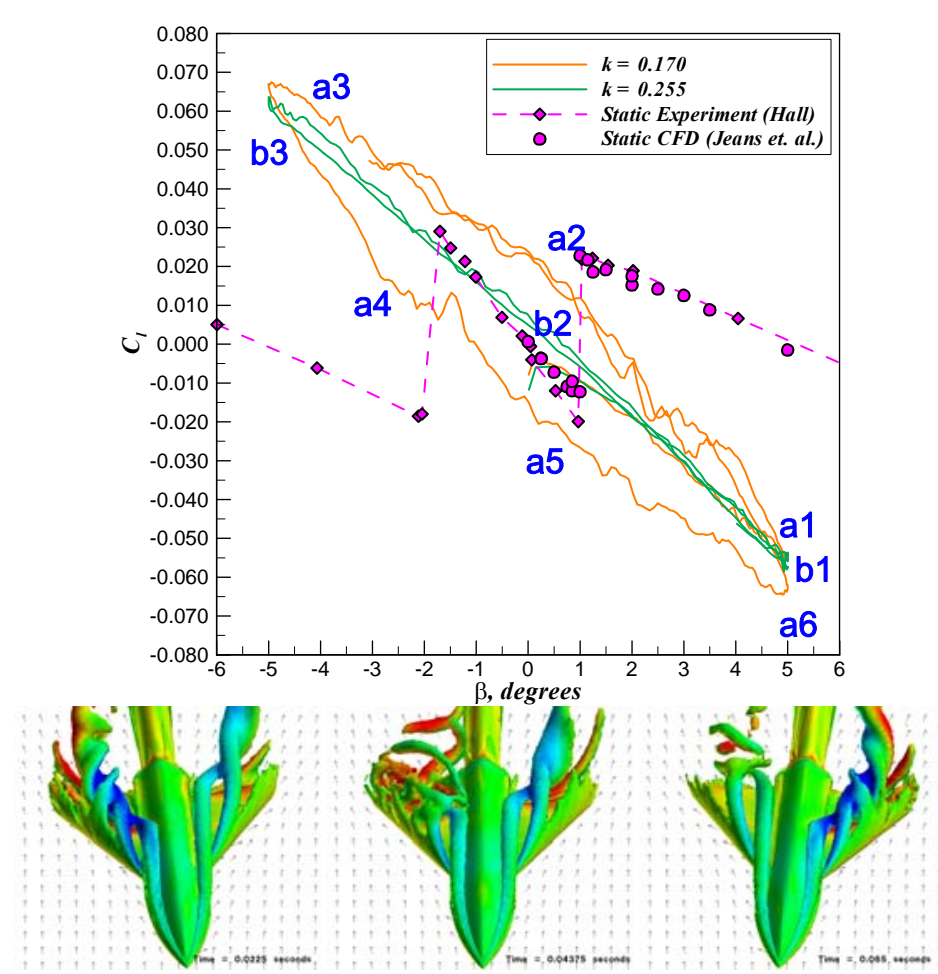

a1

a2

a3

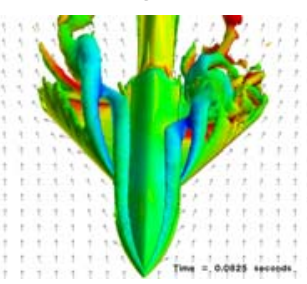

a4

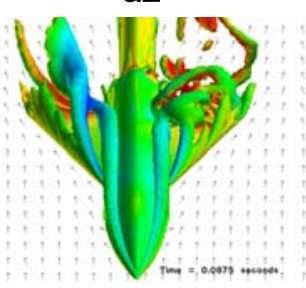

a5

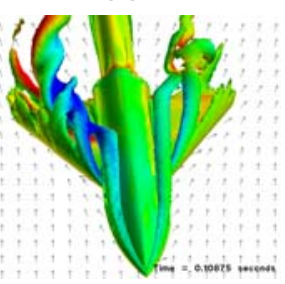

a6

(a) $k=0.17$.

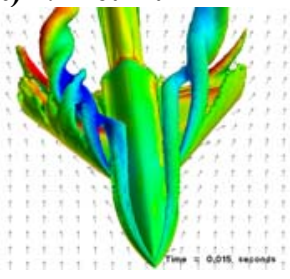

b1

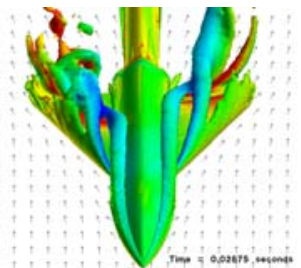

b2

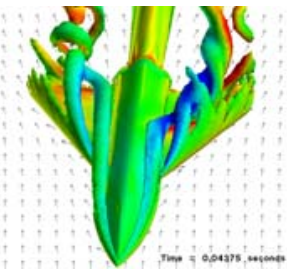

b3 (b) $k=0.255$.

Figure 11. Isosurfaces of $Q=1.0 \times 10^{7} \mathrm{~s}^{-2}$ at selected frames for high frequency simulations at $k=0.17$ and $k=0.255$. Isosurfaces are colored by axial velocity with red corresponding to $u=0$ and blue corresponding to $u=-2 u_{\infty}$. 

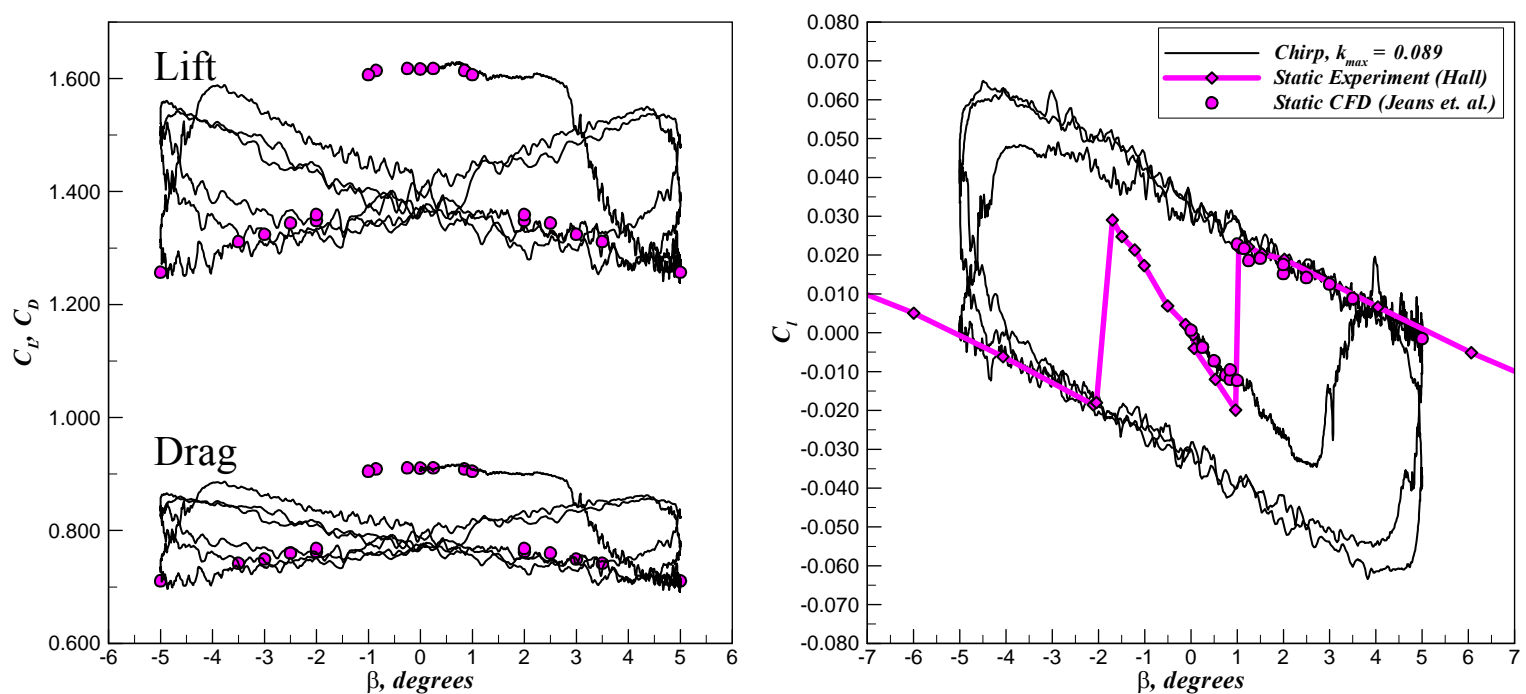

(a) Lift and drag coefficient (see (b) for legend). (b) Rolling moment coefficient.

Figure 12. CFD predicted dynamic lift, drag, and rolling moment coefficient as a function of sideslip angle for the chirp training maneuver at $\alpha=30^{\circ}, M_{\infty}=0.4$, and $\operatorname{Re}_{\bar{c}}=2.68 \times 10^{6}$.

\section{Aerodynamic Loads Modeling}

SIDPAC was used to develop S\&C models for rolling moment for each of the dynamic simulations. Required inputs into SIDPAC include the independent variables that will be used to determine the functional relationship between the aircraft motion and the computed aerodynamic loads. Based on the flow field physics, $\beta, p, \dot{p}, r$, and $\dot{r}$ were chosen as the independent variables for all of the S\&C models. One must also choose the maximum regressor order for each of the independent variables and the maximum allowable model term order. It was determined using an iterative process that setting each parameter to 4 was sufficient for the given problem. In addition, model terms that increased the coefficient of determination, $R^{2}$, by less than 0.1 percent were omitted from the model.

Table 1. Coefficients for constant frequency models.

\begin{tabular}{|c|c|c|c|c|c|}
\cline { 2 - 6 } \multicolumn{1}{c|}{} & \multicolumn{6}{c|}{ Reduced Frequency $(\boldsymbol{k})$} \\
\cline { 2 - 6 } \multicolumn{1}{c|}{} & $\mathbf{0 . 2 5 5}$ & $\mathbf{0 . 1 7 0}$ & $\mathbf{0 . 0 8 5}$ & $\mathbf{0 . 0 4 2}$ & $\mathbf{0 . 0 2 1}$ \\
\hline $\mathbf{C}_{\mathbf{1}}$ & $4.041 \mathrm{E}-03$ & $4.522 \mathrm{E}-03$ & 0 & 0 & 0 \\
\hline $\mathbf{C}_{\mathbf{2}}$ & $-1.186 \mathrm{E}-02$ & $-1.255 \mathrm{E}-02$ & $-4.846 \mathrm{E}-01$ & 0 & 0 \\
\hline $\mathbf{C}_{\mathbf{3}}$ & 0 & $-7.749 \mathrm{E}-05$ & $-2.817 \mathrm{E}-04$ & $-5.938 \mathrm{E}-04$ & $-6.017 \mathrm{E}-01$ \\
\hline $\mathbf{C}_{\mathbf{4}}$ & 0 & $5.055 \mathrm{E}-12$ & 0 & $-4.572 \mathrm{E}-09$ & 0 \\
\hline $\mathbf{C}_{\mathbf{5}}$ & 0 & 0 & $-3.724 \mathrm{E}-11$ & 0 & 0 \\
\hline $\mathbf{C}_{\mathbf{6}}$ & 0 & 0 & $-7.437 \mathrm{E}-04$ & 0 & 0 \\
\hline $\mathbf{C}_{\mathbf{7}}$ & 0 & 0 & $-5.829 \mathrm{E}-12$ & 0 & 0 \\
\hline $\mathbf{C}_{\mathbf{8}}$ & 0 & 0 & 0 & $1.313 \mathrm{E}-11$ & $-5.570 \mathrm{E}-07$ \\
\hline $\mathbf{C}_{\mathbf{9}}$ & 0 & 0 & 0 & $7.759 \mathrm{E}-08$ & 0 \\
\hline $\mathbf{C}_{\mathbf{1 0}}$ & 0 & 0 & 0 & $1.651 \mathrm{E}-06$ & 0 \\
\hline $\mathbf{C}_{\mathbf{1 1}}$ & 0 & 0 & 0 & $4.464 \mathrm{E}-08$ & 0 \\
\hline $\mathbf{C}_{\mathbf{1 2}}$ & 0 & 0 & 0 & $-4.707 \mathrm{E}-05$ & 0 \\
\hline $\mathbf{C}_{\mathbf{1 3}}$ & 0 & 0 & 0 & $-7.546 \mathrm{E}-03$ & 0 \\
\hline $\mathbf{C}_{\mathbf{1 4}}$ & 0 & 0 & 0 & 0 & $2.772 \mathrm{E}-07$ \\
\hline $\mathbf{C}_{\mathbf{1 5}}$ & 0 & 0 & 0 & 0 & $-3.466 \mathrm{E}-01$ \\
\hline $\mathbf{C}_{\mathbf{1 6}}$ & 0 & 0 & 0 & 0 & $1.619 \mathrm{E}-08$ \\
\hline $\mathbf{C}_{\mathbf{1 7}}$ & 0 & 0 & 0 & 0 & $-5.012 \mathrm{E}-10$ \\
\hline $\mathbf{C}_{\mathbf{1 8}}$ & 0 & 0 & 0 & 0 & $-3.209 \mathrm{E}-07$ \\
\hline
\end{tabular}

\section{Constant Frequency Models}

To determine if SIDPAC can properly model the extreme nonlinear aerodynamic behavior of rolling moment with sideslip angle, $\mathrm{S} \& \mathrm{C}$ models are developed for each of the constant frequency simulations. This results in the following generic model,

$$
\begin{aligned}
& C_{l}(\beta, p, \dot{p}, r, \dot{r})=C_{1}+C_{2} \beta+C_{3} p+C_{4} \dot{r} r^{2}+C_{5} \dot{p}^{2} p+C_{6} \dot{p}+C_{7} \dot{p} p^{3}+C_{8} \dot{p}^{3}+C_{9} r \dot{p}^{2}+ \\
& C_{10} p^{2}+C_{11} \dot{r}^{2} p+C_{12} \dot{p} p+C_{13} p \beta+C_{14} \dot{p} p^{2}+C_{15} r+C_{16} p^{4}+C_{17} \dot{p}^{2} p^{2}+C_{18} \dot{r} \dot{p}^{2}
\end{aligned},
$$


where the coefficients for each term of the model is given in Table 1 for each frequency. As seen in Table 1, each model does not have eighteen terms, for example, at $k=0.17$ the model has two terms and is only dependent on sideslip angle. In general, the model increases in complexity as the frequency is reduced and the number of model terms increases from two at $k=0.17$ to seven at $k=0.021$. In addition, at higher frequencies there is a stronger dependence on sideslip angle, whereas, at lower frequencies the model is strongly dependent on roll and yaw rates. Surprisingly, at $k=0.021$ the model is completely independent of sideslip angle.

Each of the constant frequency rolling moment models is validated in Fig. 13 using the CFD data. Overall the SIDPAC models compare well with the CFD data and in general the agreement improves with increased frequency. Also shown in Fig. 13 is the $R^{2}$ value for each model, which varied from $R^{2}=0.861$ at $k=0.021$ to $R^{2}=0.997$ at $k=$ 0.255 .
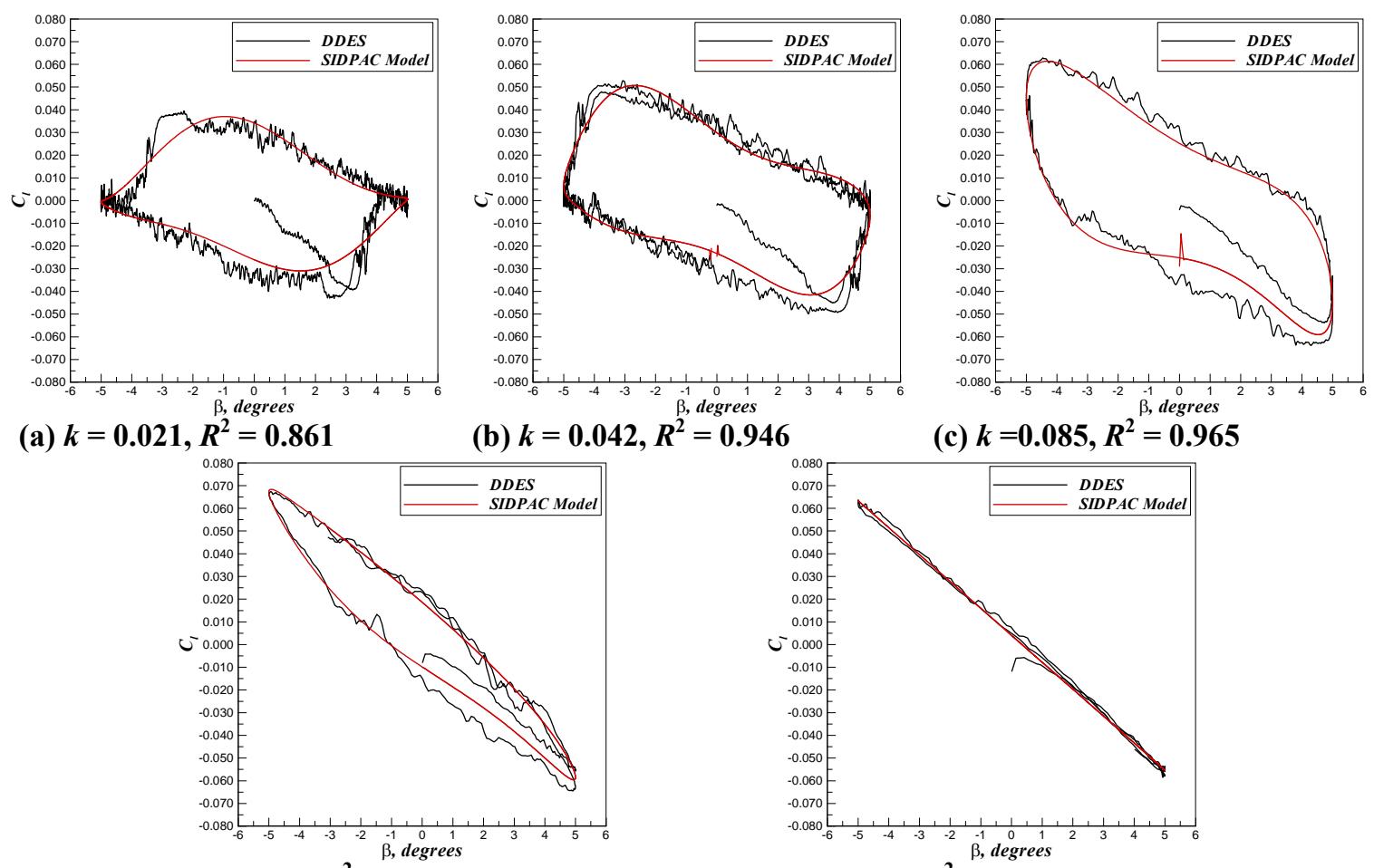

(c) $k=0.085, R^{2}=0.965$

(d) $k=0.17, R^{2}=0.988$

validation of SIDPA simulations.
One can also see in Fig. 13 that the SIDPAC models are less accurate at the lower frequencies during the upstroke from $\beta=0^{\circ}$ to $\beta=5$. This is because for this range of sideslip angle the rolling moment data is not time invariant and therefore violates the SIDPAC requirements. That is, from $\beta=0^{\circ}$ to $\beta=5^{\circ}$ there are two physically correct values of rolling moment, but the independent variables are identical. This apparent ambiguity arises due to the nature of the vortex breakdown described in Section IV B. To determine if the SIDPAC model would improve if the system were time invariant, the initial motion from $\beta=0^{\circ}$ to $\beta$ $=4^{\circ}$ is removed from the training data at $k=$ $0.0021,0.042$, and 0.085 , and new SIDPAC models are generated. The updated generic model at these frequencies is,
Table 2. Coefficients for constant frequency models with the initial motion from $\beta=0^{\circ}$ to $\beta=4^{\circ}$ excluded from the training data.

\begin{tabular}{|c|c|c|c|}
\cline { 2 - 4 } \multicolumn{1}{c|}{} & \multicolumn{3}{c|}{ Reduced Frequency $(\boldsymbol{k})$} \\
\cline { 2 - 4 } \multicolumn{1}{c|}{} & $\mathbf{0 . 0 8 5}$ & $\mathbf{0 . 0 4 2}$ & $\mathbf{0 . 0 2 1}$ \\
\hline $\mathbf{C}_{\mathbf{1}}$ & $-6.896 \mathrm{E}-03$ & 0 & 0 \\
\hline $\mathbf{C}_{\mathbf{2}}$ & $-3.354 \mathrm{E}-04$ & $-7.372 \mathrm{E}-04$ & $-7.964 \mathrm{E}-01$ \\
\hline $\mathbf{C}_{\mathbf{3}}$ & $1.964 \mathrm{E}-11$ & 0 & 0 \\
\hline $\mathbf{C}_{\mathbf{4}}$ & $-8.070 \mathrm{E}-05$ & 0 & 0 \\
\hline $\mathbf{C}_{\mathbf{5}}$ & 0 & $-3.060 \mathrm{E}-10$ & 0 \\
\hline $\mathbf{C}_{\mathbf{6}}$ & 0 & $1.117 \mathrm{E}-11$ & $-5.309 \mathrm{E}-07$ \\
\hline $\mathbf{C}_{\mathbf{7}}$ & 0 & $-1.470 \mathrm{E}-06$ & 0 \\
\hline $\mathbf{C}_{\mathbf{8}}$ & 0 & 0 & $-4.194 \mathrm{E}-05$ \\
\hline $\mathbf{C}_{\mathbf{9}}$ & 0 & 0 & $-4.589 \mathrm{E}-01$ \\
\hline $\mathbf{C}_{\mathbf{1 0}}$ & 0 & 0 & $2.120 \mathrm{E}-02$ \\
\hline
\end{tabular}

12

American Institute of Aeronautics and Astronautics DISTRIBUTION A. Approved for public release, distribution unlimited. 


$$
C_{l}(\beta, p, \dot{p}, r, \dot{r})=C_{1} \beta+C_{2} p+C_{3} \dot{p}^{2}+C_{4} \beta^{3}+C_{5} \dot{p}^{2} p+C_{6} \dot{p}^{3}+C_{7} r^{2} \beta+C_{8} \dot{p} p^{2}+C_{9} r+C_{10} \dot{p},
$$

where the coefficients for each term of the model is given in Table 2 for each frequency. Although the model is different from the previous model given in Eq. (4), many of the same general trends apply. That is, at $k=0.085$ the model is still strongly dependent on sideslip angle and as the frequency is reduced to $k=0.021$, the model transitions such that it is primarily dependent on roll rate.

The updated constant frequency rolling moment models are validated in Fig. 14 using the CFD data. Overall the SIDPAC models are in better agreement with the CFD data, with the largest improvements occurring at the lowest frequencies. At $k=0.021 R^{2}$ increases by 7.5 percent.

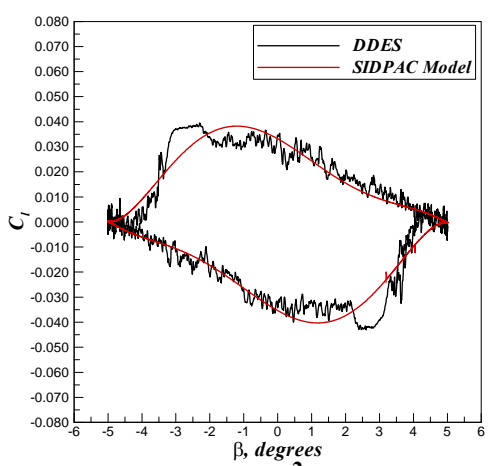

(a) $k=0.021, R^{2}=0.935$

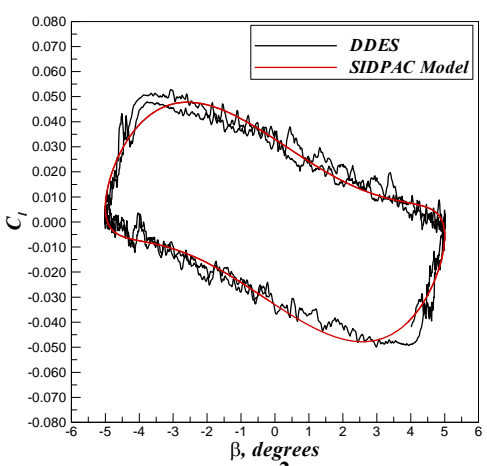

(b) $k=0.042, R^{2}=0.971$

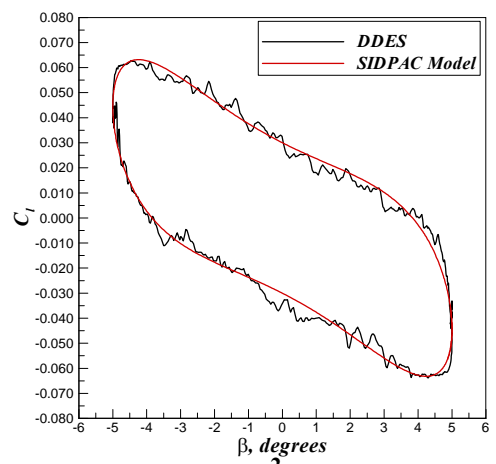

(c) $k=0.085, R^{2}=0.992$

Figure 14. Validation of SIDPAC based rolling moment models to CFD predicted at $k=0.021,0.042$, and 0.085 with the initial motion from $\beta=0^{\circ}$ to $\beta=4^{\circ}$ excluded from the training data.

One may also argue that since the system is not time invariant the proper independent variables were not chosen for the SIDPAC model and this issue could be resolved by properly choosing these variables. One possible choice might be to include the time history of a single surface pressure reading that is known to exhibit a time varying behavior similar to the rolling moment data. Plotted in Fig. 15 (a) is the time history of a pressure reading on the top of the aircraft + -axis wing for $k=0.085$. Including this reading as an independent variable in the SIDPAC model results in the follow aerodynamic model for rolling moment,

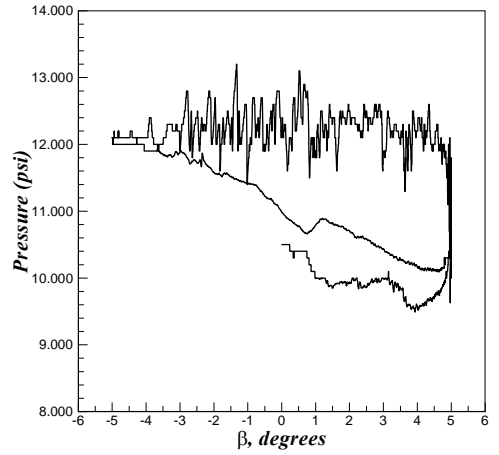

(a)

Figure 15. (a) Time history of a single pressure reading on the $+y-$ axis wing for $k=0.085$. (b) Validation of SIDPAC model to CFD data when the surface pressure reading is used as an independent variable.

$$
\begin{aligned}
& C_{l}\left(P_{1} \beta, p, \dot{p}, r, \dot{r}\right)=C_{1}+C_{2} P_{1}+C_{3} P_{1}^{4}+C_{4} p^{3} P_{1}+ \\
& C_{5} \dot{p} P_{1}^{2}+C_{6} \dot{r}^{2} r+C_{7} \beta+C_{8} r \beta^{3}+C_{9} \dot{p} p^{2}+C_{10} \beta^{4}+C_{11} p \beta
\end{aligned},
$$

where $P_{1}$ is the surface pressure reading. The updated rolling moment model is validated in Fig. 15 (b) using the CFD data. Including the surface pressure reading clearly improves the model predictions for sideslip angles between $\beta=0^{\circ}$ and $\beta=4^{\circ}$, and increases $R^{2}$ by 2.5 percent. Presumably, increasing the number of time varying independent variables (e.g. multiple surface pressure readings) would result in further improvements. 


\section{Chirp Motion Model}

A final SIDPAC model is generated using the chirp motion as the training maneuver. The goal of this model is to reliably interpolate the rolling moment response for all frequencies bound by the maximum and minimum frequency of the chip motion. Using the guidelines outlined at the beginning of this section results in the following six term model for rolling moment,

$$
C_{l}(\beta, p, \dot{p}, r, \dot{r})=C_{1} p+C_{2} \dot{p}+C_{3} r^{3}+C_{4} \beta^{3} C_{5} \beta+C_{6} \dot{r} r^{2}
$$

This model is validated in Fig. 16 using the CFD data for the chirp maneuver. Overall the SIDPAC model compares well with the CFD data with the largest discrepancies again occur at the lower frequencies. This is most likely due to the highly nonlinear aerodynamic behavior at these frequencies. The coefficient of determination for this model is $R^{2}=0.946$.

Ideally the SIDPAC model developed using the chirp training maneuver can be used to predict the rolling moment at any frequency bound within the maneuver. To test this hypothesis, the aerodynamic model defined in Eq. (7) is used to predict the constant frequency rolling moment at $k=0.021,0.042$, and 0.085 . The model predictions are compared to the CFD data in Fig. 17. Overall the results are very encouraging, and demonstrate the model's ability to predict the rolling moment response at a variety of frequencies. Based on this result it is reasonable to assume this aerodynamic model could reliably interpolate to any frequency between $f=1.43$ and 5.86 Hertz, which could potentially result in huge time and CPU hours savings. For the current case all three constant frequency simulations combined for approximately $118 \mathrm{~K}$ CPU hours, while the chirp simulation required $68 \mathrm{~K} \mathrm{CPU}$ hours and contains all of the frequency content between the constant frequency simulations.

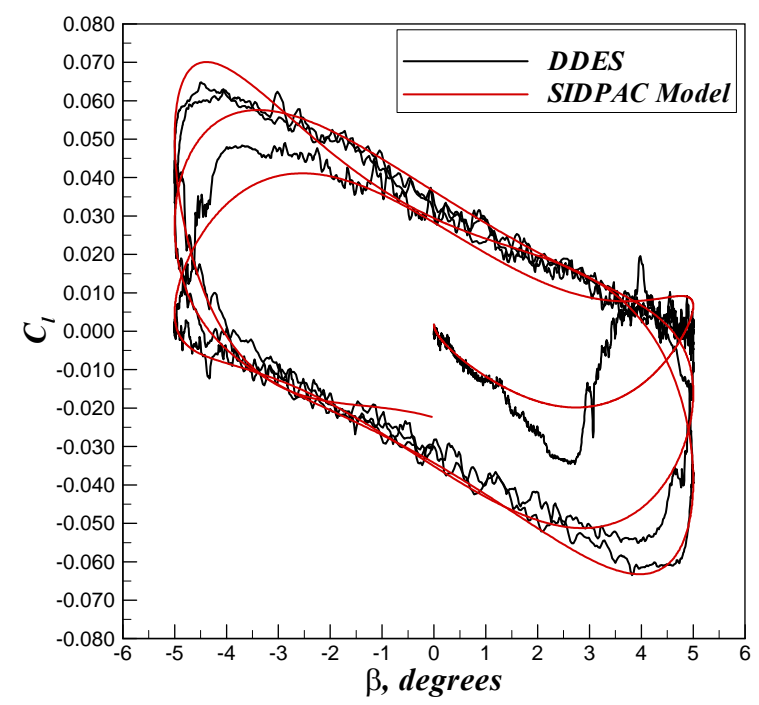

Figure 16. Validation of SIDPAC based rolling moment model to CFD predicted for chip motion with reduced frequency varying linearly from $k=0$ to $k=$ 0.089.

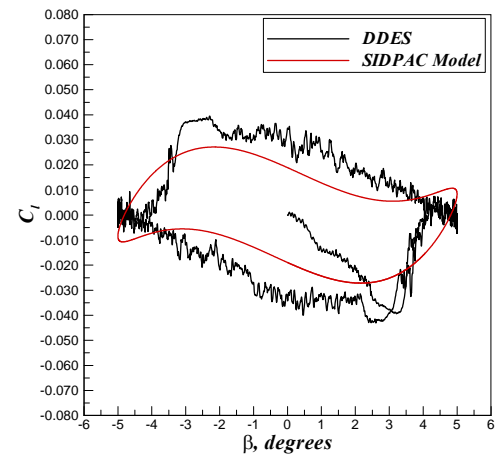

(a) $k=0.021$

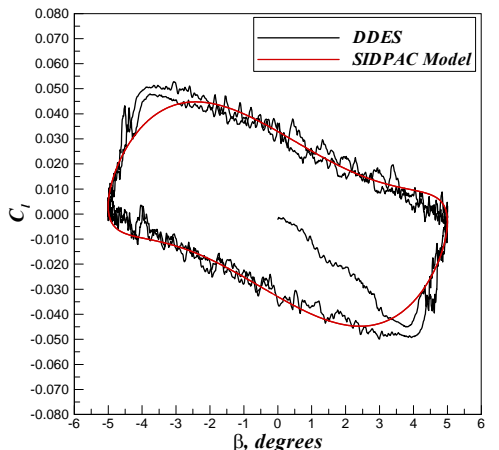

(b) $k=0.042$

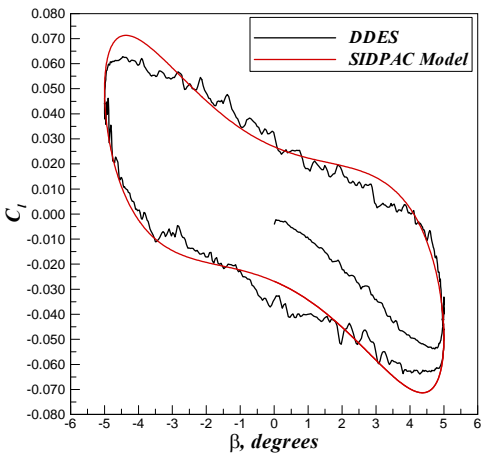

(c) $k=0.085$

Figure 17. Predicted rolling moment response for constant frequency motions at $k=0.021,0.042$, and 0.085 using the model that was trained with the chirp maneuver.

Theoretically, the chirp maneuver contains reduced frequency content ranging from $k=0$ to $k=0.089$, therefore it may be capable of predicting rolling moment responses at frequencies less than $k=0.021$. Plotted in Fig. 18 are the chirp based model predictions at $k=0.0075,0.00075$ and 0.000075 , which correspond to frequencies of $f=0.5$, 0.05 and 0.005 Hertz, respectively. A comparison of the model predictions to the static data shows that improvements are necessary for a good quantitative prediction at these extremely low frequencies. However, the

14

American Institute of Aeronautics and Astronautics DISTRIBUTION A. Approved for public release, distribution unlimited. 
SIDPAC model does give a qualitative indication that unwanted aerodynamic behavior is occurring, and therefore would raise a red flag for a $\mathrm{S} \& \mathrm{C}$ engineer during the design phase, which is a promising result. A possible reason for the discrepancy is that the static data must only be dependent on sideslip angle and the initial condition, however, all of the constant frequency models show that as frequency is decreased the dependency on sideslip angle is reduced while the dependency on roll and yaw rate is increased. As some frequency the flow must transition such that it is again strongly dependent on sideslip angle, however it is likely that this frequency is very low and there may be insufficient training data at these lower frequencies to accurately model this behavior.

\section{Conclusion}

Dynamic Delayed Detached-Eddy Simulations were performed on the MTVI configuration at a Reynolds number of 2.68 million, Mach number of 0.4 , and angle of incidence of $30^{\circ}$, while sideslip was varied in a sinusoidal manner from $+/-5.0^{\circ}$. It was verified that the strongly nonlinear rolling moment behavior that was experimentally measured and computationally predicted for the static case, also exists in the dynamic case. Key differences in the static and dynamic nonlinearity were also highlighted. The CFD results have confirmed that this nonlinear behavior is primarily due to abrupt asymmetric vortex breakdown over the windward wing. In general, the severity of this nonlinearity reduced with increasing frequency and was completely removed at a frequency of 17.1 Hertz.

The authors have also shown that it is feasible to use high quality CFD simulations as training maneuvers to develop SIDPAC based lower-order aerodynamic loads models for aircraft configurations with severely nonlinear aerodynamic behavior. Reliable aerodynamic models were developed for rolling moment for a variety of frequencies ranging from 1.43 to $17.1 \mathrm{Hertz}$. In addition, an aerodynamic model trained using a varying frequency chirp maneuver was capable of predicting highly nonlinear behavior at constant frequencies ranging from 1.43 to 5.85 Hertz. Such predictive capabilities will improve the design of future fighters and could drastically reduce the cost associated with flight testing new or modified aircraft.

\section{Acknowledgments}

The authors would like to thank the Air Force Office of Scientific Research, the United States Air Force Academy Modeling and Simulation Research Center, and Dr. Scott A. Morton from the United States Air Force Seek Eagle Office for their generous financial support throughout this project. The authors would also like to thank Dr. Robert M. Hall of the NASA Langley Research Center for providing the geometry and wind tunnel data.

\section{References}

${ }^{1}$ McDaniel, D.R., Cummings, R.M., Bergeron, K., Morton, S.M, Dean, J.P., "Comparisons of CFD Solutions of Static and Maneuvering Fighter Aircraft with Flight Test Data," $3^{\text {rd }}$ International Symposium on Integrating CFD and Experiments in Aerodynamics, 20-21 June 2007, US Air Force Academy, CO.

${ }^{2}$ Yurkovich, R., and Chen, P.C., "State-of-the-Art of Unsteady Aerodynamics for High Performance Aircraft," AIAA-20010428, January 2001.

${ }^{3}$ Meyn, L.A., and James, K.D., "Full Scale Wind Tunnel Studies of F/A-18 Tail Buffet,” Journal of Aircraft, Vol. 33, No. 3, pp. 589-595, 1996.

${ }^{4}$ Jacobson, S.B., Britt, R.T., Freim, D.R., and Kelly, P.D., "Residual Pitch Oscillation Flight Test Analysis on the B-2 Bomber," AIAA 1998-1805, January 1998.

${ }^{5}$ Forsythe, J.R., Hoffmann, K.A., Cummings, R.M., and Squires, K.D., "Detached-Eddy Simulation with Compressibility Corrections Applied to a Supersonic Axisymmetric Base," Journal of Fluids Engineering, Vol. 124, No. 4, 2002 , pp. 911-923.

${ }^{6}$ Forsythe, J.R., and Woodson, S.H., "Unsteady Computations of Abrupt Wing Stall Using Detached-Eddy Simulation," Journal of Aircraft, Vol. 42, No. 3, 2005, pp. 606-616.

\section{5}

American Institute of Aeronautics and Astronautics

DISTRIBUTION A. Approved for public release, distribution unlimited. 
${ }^{7}$ Morton, S.A., Forsythe, J.R., Mitchell, A.M., and Hajek, D., "Detached-Eddy Simulations and Reynolds-Averaged NavierStokes Simulations of Delta Wing Vortical Flowfields," Journal of Fluids Engineering, Vol. 124, No. 4, 2002 , pp. $924-932$.

${ }^{8}$ J. R. Forsythe, K. D. Squires, K. E. Wurtzler, and P. R. Spalart, "Detached-Eddy Simulation of Fighter Aircraft at High Alpha," Journal of Aircraft, Vol. 41, No. 2, pp. 193-200, 2004.

${ }^{9}$ J. R. Forsythe, C. M. Fremaux, and R. M. Hall, "Calculation of Static and Dynamic Stability Derivatives of the F/A-18E in Abrupt Wing Stall Using RANS and DES," in Proceedings of International Conference for CFD, Toronto, Canada, 2004.

${ }^{10}$ M. Claus, S. A. Morton, and R. Cummings, "DES Turbulence Modelling on the C-130 Comparison Between Computational and Experimental Results," AIAA Paper 2005-884, 2005.

${ }^{11}$ S. A. Morton, and J. R. Forsythe, K. D. Squires, R. M. Cummings, "Detached-Eddy Simulations of Full Aircraft Experiencing Massively Separated Flows,” Computational Fluid Dynamics Journal ISCFD Japan, Vol. 13, No. 3, January, 2005.

${ }^{12}$ Jeans, T.J., McDaniel, D.R., Cummings, R.M., and Mason, W.H., "Aerodynamic Analysis of a Generic Fighter with a Chine Fuselage/Delta Wing Configuration Using Delayed Detached-Eddy Simulation,”, AIAA $26^{\text {th }}$ Applied Aerodynamics Conference, accepted, August 2008.

${ }^{13}$ Matthews, L.L., and Schwartz, M.S., "Modeling and Simulations of a Dynamic Maneuvering F-16," 2008 AIAA Student Conference, May 2008.

${ }^{14}$ Görtez, Stefan, and McDaniel, David, "Towards an Efficient Aircraft Stability and Control Analysis Capability using HighFidelity CFD," 45 ${ }^{\text {th }}$ AIAA Aerospace Sciences Meeting and Exhibit, AIAA Paper 2007-1053, 2007.

${ }^{15}$ Rao, D.M., and Bhat, M.K., "A Low-Speed Wind Tunnel Study of Vortex Interaction Control Techniques on a ChineForebody/Delta-Wing Configuration,” NASA Contractor Report, NASA-CR-189616, March 1992.

${ }^{16}$ Rao, D.M., and Bhat, M.K., "Subsonic Investigations of Vortex Interaction Control For Enhanced High-Alpha Aerodynamics of a Chine Forebody/ Delta Wing Configuration," NASA Contractor Report, NASA-CR-189641, June 1992.

${ }^{17}$ Rao, D.M., and Bhat, M.K., "High-Alpha Vortex Decoupling Investigations on a Chine Forebody/Delta Wing Configuration at Transonic Mach Numbers," NASA Contractor Report, NASA-CR-189642, June 1992.

${ }^{18}$ Hall, R.M., "Impact of Fuselage Cross Section on the Stability of a Generic Fighter," $16^{\text {th }}$ Applied Aerodynamics Conference, AIAA-98-2725, 1998.

${ }^{19}$ Ghaffari, F., “ On the Vortical-Flow Prediction Capability of an Unstructured-Grid Euler Solver," 32 nd Aerospace Sciences Meeting and Exhibit, AIAA-94-0163, January 1994.

${ }^{20}$ Kinard, T. A., Harris, B. W., and Raj, P., "Computational Simulation of Benign and Burst Vortex Flows," $13^{\text {th }}$ AIAA Applied Aerodynamics Conference, AIAA-95-1815-CP, June 1995.

${ }^{21}$ Finley, D.B., and Karman S.L. Jr., "Application of Splitflow Unstructured CFD Code to Euler Predictions of a Generic Fighter Model," $13^{\text {th }}$ AIAA Applied Aerodynamics Conference, AIAA-95-1821-CP, June 1995.

${ }^{22}$ Finley, D.B., "Euler Technology Assessment Program for Preliminary Aircraft Design Employing SPLITFLOW Code With Cartesian Unstructured Grid Method," NASA Contractor Report, NASA-CR-4649, March 1995.

${ }^{23}$ Kinard, T.A., Harris, B.W., and Raj, P., "An Assessment of Viscous Effects in Computational Simulation of Benign and Burst Vortex Flows on Generic Fighter ‘wind-Tunnel Models Using TEAM Code,” NASA Contractor Report, NASA-CR-4650, March 1995.

${ }^{24}$ Treiber, D.A., and Muilenburg D.A., "Euler Technology Assessment for Preliminary Aircraft Design Employing OVERFLOW Code With Multiblock Structured-Grid Method," NASA Contractor Report, NASA-CR-4651, March 1995.

${ }^{25}$ Kinard, T.A., and Raj, P., "Euler Technology Assessment for Preliminary Aircraft Design - Compressibility Predictions by Employing the Unstructured Grid USM3D Code," NASA Contractor Report, NASA-CR-4711, March 1996.

${ }^{26}$ Pirzadeh, S.Z., "A Solution-Adaptive Unstructured Grid Method by Grid Subdivision and Local Remeshing," Journal of Aircraft, Vol.37, No. 5, September-October 2000.

${ }^{27}$ Raj, P., Finley, D.B., and Ghaffari, F., "An Assessment of CFD Effectiveness for Vortex-Flow Simulation to Meet Preliminary Design Needs Part A - Vortex Flows and High Angle of Attack," Paper No. 47, NATO/RTO/AVT-Panel, May 2001.

${ }^{28}$ Strang, W.Z., Tomaro, R.F., Grismer, M.J., "The Defining Methods of Cobalt60: A Parallel, Implicit, Unstructured Euler/Navier Stokes Flow Solver," $37^{\text {th }}$ AIAA Aerospace Sciences Meeting and Exhibit, AIAA Paper 99-0786, January 1999.

${ }^{29}$ Gottlieb, J.J., and Groth, C.P.T., “Assessment of Riemann Solvers for Unsteady One-Dimensional Inviscid Flows of Perfect Gasses," Journal of Computation Physics, Vol. 78, No.2, 1988, pp. 437-458.

${ }^{30}$ Tomaro, R.F., Strang, W.Z., Sankar, L.N., "An Implicit Algorithm for Solving Time Dependent Flows On Unstructured Grids," $35^{\text {th }}$ AIAA Aerospace Sciences Meeting \& Exhibit, AIAA Paper 97-0333, January 1999.

${ }^{31}$ Spalart P.R., Deck S., Shur M.L., Squires K.D., Strelets, M.Kh., and Travin, A., "A New Version of Detached-Eddy Simulation, Resistant to Ambiguous Grid Densities," Theoretical Computational Fluid Dynamics, Vol. 20, 2006, pg. 181-195.

${ }^{32}$ Spalart, P.R., Jou, W.H., Strelets, M. and Allmaras, S.R., "Comments on the Feasibility of LES for Wings, and on a Hybrid RANS/LES approach,” Advances in DNS/LES, $1^{\text {st }}$ AFOSR International Conference on DNS/LES, Greyden Press, Columbus. OH, 1997.

${ }^{33}$ Spalart, P.R., and Allmaras, S.R., "A One Equation Turbulence Model for Aerodynamic Flows," La Recherche Aerospatiale, Vol. 1, 1994, pp. 5-21.

${ }^{34}$ Menter, F.R., Kuntz, M., "Adaptation of Eddy-Viscosity Turbulence Models to Unsteady separated Flow Behind Vehicles," In: McCallen, R., Browand, F., Ross, J. (eds.) Symposium on "the aerodynamics of heavy vehicles: trucks, busses and trains." Monterey, USA, 2-6 Dec 2002, Springer, Berlin Heidelberg NewYork, 2004.

${ }^{35}$ Shur, M.L., Strelets, M.K., Travin, A.K., Spalart, P.R., "Turbulence Modeling in Rotating and Curved Channels: Assessing the Spalart-Shur Correction," AIAA Journal, Vol. 38, No. 5, May 2000.

16

American Institute of Aeronautics and Astronautics

DISTRIBUTION A. Approved for public release, distribution unlimited. 
${ }^{36}$ Cobalt V4.0, www.cobaltcfd.com, June 2008. 2008.

${ }^{37}$ Mississippi State University Computational Simulation and Design Center, "Solid Mesh 5.45 3D Users Manual," July

${ }^{38}$ Cummings, R.M., Morton, S.A., McDaniel D.R., "Experiences in Accurately Predicting Time-Dependant Flows," Progress in Aerospace Sciences, Vol. 44, 2008, pp. 241-257.

${ }^{39}$ Ljung, L., "System Identification. Theory for the User," Prentice Hall Information and System Sciences Series.

${ }^{40}$ Klein, V. and Morelli, E.A., "Aircraft System Identification - Theory and Practice," AIAA Educational Series, 2006.

${ }^{41}$ Morelli, E.A., "System IDentification Programs for AirCraft (SIDPAC)," AIAA Paper 2002-4704, 2002.

${ }^{42}$ Dubief Y., and Delcayre, F., "On Coherent-Vortex Identification in Turbulence," Journal of Turbulence, Vol. 1, pg. 1-22, 2000. 